\title{
A!
}

This is an electronic reprint of the original article.

This reprint may differ from the original in pagination and typographic detail.

Hannonen, Antti; Shevchenko, Andriy; Friberg, Ari; Setälä, Tero

\section{Temporal phase-contrast ghost imaging}

Published in:

Physical Review A

DOI:

10.1103/PhysRevA.102.063524

Published: 17/12/2020

Document Version

Publisher's PDF, also known as Version of record

Please cite the original version:

Hannonen, A., Shevchenko, A., Friberg, A., \& Setälä, T. (2020). Temporal phase-contrast ghost imaging.

Physical Review A, 102(6), [063524]. https://doi.org/10.1103/PhysRevA.102.063524

This material is protected by copyright and other intellectual property rights, and duplication or sale of all or part of any of the repository collections is not permitted, except that material may be duplicated by you for your research use or educational purposes in electronic or print form. You must obtain permission for any other use. Electronic or print copies may not be offered, whether for sale or otherwise to anyone who is not an authorised user. 


\title{
Temporal phase-contrast ghost imaging
}

\author{
Antti Hannonen $\odot,{ }^{1,}{ }^{*}$ Andriy Shevchenko, ${ }^{2}$ Ari T. Friberg, ${ }^{1}$ and Tero Setälä ${ }^{1}$ \\ ${ }^{1}$ Institute of Photonics, University of Eastern Finland, P.O. Box 111, FI-80101 Joensuu, Finland \\ ${ }^{2}$ Department of Applied Physics, Aalto University, P.O. Box 13500, FI-00076 Aalto, Finland
}

(Received 13 October 2020; accepted 30 November 2020; published 17 December 2020)

\begin{abstract}
We introduce a phase-contrast ghost-imaging scheme for the characterization of temporal phase objects in terms of intensity correlations at two photodetectors. The technique is analogous to Zernike's phase-contrast imaging method and is based on utilizing a suitable filter function which renders the small-amplitude phase variations visible in the intensity correlation function. The approach is insensitive to temporal distortions and offers a promising method to analyze the phases of optical pulses.
\end{abstract}

DOI: 10.1103/PhysRevA.102.063524

\section{INTRODUCTION}

Ghost imaging produces an image of an object by correlating random intensities of incoherent light at two photodetectors [1-3]. The measurement arrangement consists of two arms referred to as the test and reference arms. In the test arm, light transmitted (or reflected) by the object is measured by a bucket (or single-pixel) detector whereas in the reference arm, the same random intensity pattern but without interaction with the object is recorded by a detector with resolution. Neither of the detectors alone can characterize the object whose image is nonetheless included in the correlation of intensity fluctuations. The technique has particular potential for imaging under turbulent [4-7] or low-light-level conditions [8] as well as in harsh environments where conventional imaging methods are hard or impossible to implement [9]. Recently, ghost imaging has been demonstrated in such diverse applications as x-ray [10], atom [11], and neutron [12] imaging, as well as in encryption [13], spectroscopy [14], ellipsometry $[15,16]$, and polarimetry using Stokes correlations [17].

Besides the spatial domain, ghost imaging of temporal objects has also been considered both theoretically $[18,19]$ and experimentally [20,21]. Temporal ghost imaging is implemented using an optical fiber-based system with the spatially incoherent source replaced by a temporally incoherent source. The temporal correlation measurements have been applied to image the temporal profiles of optical pulses even when they are severely distorted by the transmission medium [20].

In their basic forms, ghost-imaging methods are insufficient to visualize pure phase objects. For such objects in the spatial domain, a ghost-imaging analogy to Zernike's phasecontrast method [22] has been implemented [3,23-25] but imaging of temporal phase objects has not been considered so far. In this paper, we introduce a time-domain ghost-imaging arrangement that can perform phase-contrast imaging in terms of Fourier-space processing to characterize pure temporal phase objects. As with ordinary temporal ghost imaging, the

\footnotetext{
*Corresponding author: antti.hannonen@uef.fi
}

advantage of the proposed technique is its inherent insensitivity to temporal distortions. As such, the method is assumed to provide a robust means to characterize the phases of rapidly evolving waveforms distorted, e.g., by dispersive optical components.

This work is structured as follows. In Sec. II, we present the temporal ghost imaging arrangement and in Sec. III consider imaging of pure phase objects. Finally, Sec. IV summarizes the paper.

\section{TEMPORAL GHOST-IMAGING SETUP}

A random, stationary scalar light beam produced by a temporally incoherent light source with electric field $E(t)$ and obeying Gaussian statistics is guided to the ghost-imaging device shown in Fig. 1. The field propagates through a singlemode optical fiber (SMF) to a 50/50 FC where it is divided into two arms. The arms consist of four SMF sections with group-delay dispersion (GDD) parameters $\Phi_{i}=\beta_{2 i} z_{i}, i \in$ $\{a, b, c, d\}$, where $\beta_{2 i}$ denotes the group-velocity dispersion coefficient and $z_{i}$ is the propagation length. Light in the reference (upper) arm propagates to a fast photodiode detector $\left(D_{1}\right)$ through a temporal mask with transmission function $m_{1}(t)$. The test (lower) arm contains a slow photodiode detector $\left(\mathrm{D}_{2}\right)$ and a deterministic temporal object $m(t)$ placed against a temporal lens with focal length $\gamma$. The object-lens system has a combined transmission function $m_{2}(t)=m(t) \exp \left(i t^{2} / 2 \gamma\right)$. In this paper, we consider a pure phase object with $m(t)=$ $\exp \left\{i\left[\phi_{0}+\Delta \phi(t)\right]\right\}$, where $\phi_{0}$ is a constant and $|\Delta \phi(t)| \ll 2 \pi$ represents a small-amplitude phase variation. The purpose of temporal phase-contrast ghost imaging is to retrieve $\Delta \phi(t)$ using an intensity correlation measurement. The temporal phase variation induced by the object and lens can be implemented by modulating the incident light field with the help of electrooptical modulators [26,27].

Photodiode $\mathrm{D}_{1}$ detects intensity $I_{1}(t)$ and it is sufficiently fast to record the instantaneous intensity fluctuations of the incident light. The time resolution of $\mathrm{D}_{1}$ affects the resolution and visibility of the ghost image [20]. The integrating photodiode $\mathrm{D}_{2}$ is slow and cannot resolve the temporal intensity 


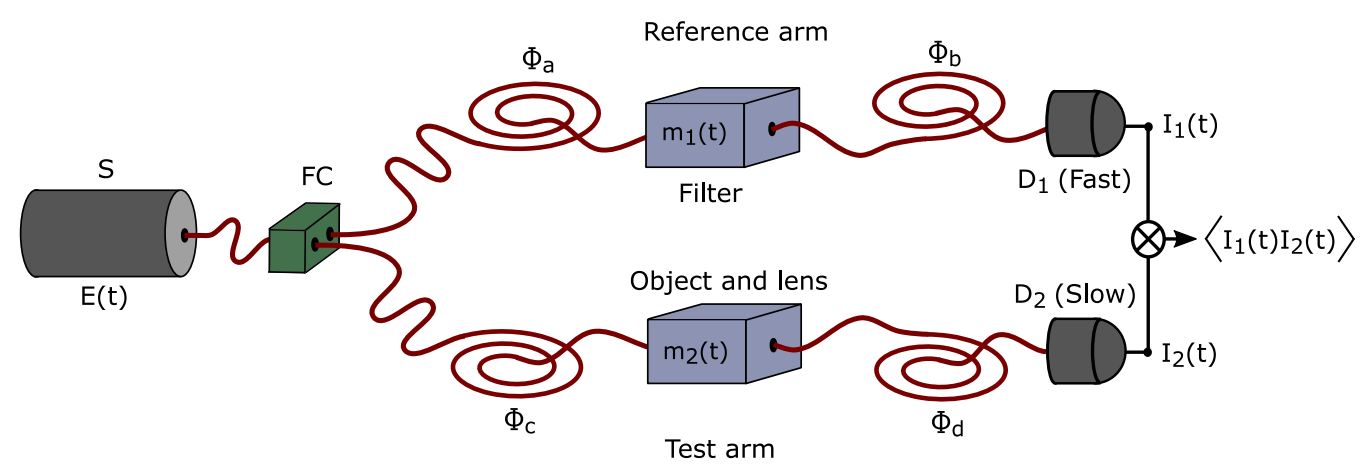

FIG. 1. Illustration of the phase-contrast ghost-imaging setup. The system consists of a temporally incoherent light source (S), 50/50 fiber coupler (FC), temporal filter $\left[m_{1}(t)\right]$, temporal lens against a temporal object $\left[m_{2}(t)\right]$, fast detector $\left(\mathrm{D}_{1}\right)$, and slow detector $\left(\mathrm{D}_{2}\right)$. The source field $E(t)$ is split into the reference and test arms consisting of four single-mode fiber sections with group-delay dispersion parameters $\Phi_{i}$, $i \in\{a, b, c, d\}$. The intensities at the arm ends are $I_{1}(t)$ and $I_{2}(t)$.

structure and it acts as a bucket detector collecting all intensity falling on it. The signal produced by $\mathrm{D}_{2}$ is sensitive to the integrated intensity $\int_{-T}^{T} I_{2}(t) d t$ where the integration extends over a suitable time interval from $-T$ to $T$ that is long compared to the object and filter durations. Recording $I_{1}(t)$ and the integrated $I_{2}(t)$ for several time intervals of equal length which contain the same (deterministic) temporal object and filter generates an ensemble of random realizations carrying object information in statistical form. The various timescales involved in the process are as follows. The response time of $D_{1}$ is less than the characteristic scale of intensity fluctuations, which is much shorter than the timescales of the object and filter. These conditions can be met in practice. The response times of the fastest photodetectors are on the order of $10 \mathrm{ps}$ and a suitable source with the intensity fluctuations on this timescale is, for example, a multimode laser (consistent with Gaussian statistics) with the bandwidth of $100 \mathrm{GHz}$ (about $0.1 \mathrm{~nm}$ ) in the visible spectral range.

From now on, we use $t_{0}, t_{1}^{\prime}$, and $t_{2}^{\prime}$ to denote the instant of time at the source, reference arm filter, and test arm object, respectively, whereas $t_{1}\left(t_{2}\right)$ will refer to time at the detector $D_{1}\left(D_{2}\right)$. The intensities measured by the two photodiodes are correlated yielding an intensity correlation function $\left\langle I_{1}\left(t_{1}\right) I_{2}\left(t_{2}\right)\right\rangle_{\text {int }}=\left\langle I_{1}\left(t_{1}\right) \int_{-T}^{T} I_{2}\left(t_{2}\right) d t_{2}\right\rangle$, where the angle brackets denote ensemble averaging. Invoking the intensity fluctuations $\Delta I_{n}\left(t_{n}\right)=I_{n}\left(t_{n}\right)-\left\langle I_{n}\left(t_{n}\right)\right\rangle, n \in\{1,2\}$, around the average values $\left\langle I_{n}\left(t_{n}\right)\right\rangle$, the intensity correlation function can be written as

$$
\begin{aligned}
\left\langle I_{1}\left(t_{1}\right) I_{2}\left(t_{2}\right)\right\rangle_{\mathrm{int}}= & \left\langle I_{1}\left(t_{1}\right)\right\rangle \int_{-T}^{T}\left\langle I_{2}\left(t_{2}\right)\right\rangle d t_{2} \\
& +\left\langle\Delta I_{1}\left(t_{1}\right) \Delta I_{2}\left(t_{2}\right)\right\rangle_{\mathrm{int}},
\end{aligned}
$$

where $\left\langle\Delta I_{1}\left(t_{1}\right) \Delta I_{2}\left(t_{2}\right)\right\rangle_{\text {int }}=\int_{-T}^{T}\left\langle\Delta I_{1}\left(t_{1}\right) \Delta I_{2}\left(t_{2}\right)\right\rangle d t_{2}$. The first quantity on the right-hand side of Eq. (1) is a constant while the second term denotes the correlation of the intensity fluctuations and contains the object information. For fields obeying Gaussian statistics, the moment theorem [28] implies that the ( $t_{2}$-integrated) correlation of intensity fluctuations can be expressed as

$$
\left\langle\Delta I_{1}\left(t_{1}\right) \Delta I_{2}\left(t_{2}\right)\right\rangle_{\text {int }}=\int_{-T}^{T}\left|\Gamma\left(t_{1}, t_{2}\right)\right|^{2} d t_{2},
$$

where

$$
\Gamma\left(t_{1}, t_{2}\right)=\left\langle E_{1}^{*}\left(t_{1}\right) E_{2}\left(t_{2}\right)\right\rangle .
$$

This quantity is the mutual coherence function between the random fields $E_{1}\left(t_{1}\right)$ and $E_{2}\left(t_{2}\right)$ incident on the reference and test arm detectors, respectively.

Propagation of light in a fiber with the GDD parameter of $\Phi$ obeys

$$
E_{\text {out }}(t)=\sqrt{\frac{i}{2 \pi \Phi}} \int_{-\infty}^{\infty} E_{\text {in }}\left(t^{\prime}\right) \exp \left[\frac{-i\left(t-t^{\prime}\right)^{2}}{2 \Phi}\right] d t^{\prime},
$$

where $E_{\text {in }}\left(t^{\prime}\right)$ and $E_{\text {out }}(t)$ are the fields at the fiber input and output. The source is band-limited and propagation in an SMF acts as a Gaussian chirp filter [29] allowing the extension of the integration limits to infinity without violating causality. The above result is mathematically similar to the Fresnel diffraction integral expressing the space-time duality $[29,30]$ and the key factor behind the analogy of spatial and temporal ghost imaging. More generally, paraxial diffraction and dispersive pulse broadening are physically similar phenomena in the sense that the equations governing these two effects are formally identical. The reference and test arms are both composed of two fiber segments. The fields at the arm ends are given by

$$
E_{i}\left(t_{i}\right)=\int_{-\infty}^{\infty} E\left(t_{0}\right) K_{i}\left(t_{i}, t_{0}\right) d t_{0}, \quad i \in\{1,2\}
$$

where the kernels corresponding to propagation in the reference and test arms are, respectively,

$$
\begin{aligned}
K_{1}\left(t_{1}, t_{0}\right)= & \frac{i}{2 \pi \sqrt{\Phi_{a} \Phi_{b}}} \int_{-\infty}^{\infty} m_{1}\left(t_{1}^{\prime}\right) \\
& \times \exp \left[-i \frac{\left(t_{1}^{\prime}-t_{0}\right)^{2}}{2 \Phi_{a}}-i \frac{\left(t_{1}-t_{1}^{\prime}\right)^{2}}{2 \Phi_{b}}\right] d t_{1}^{\prime}, \\
K_{2}\left(t_{2}, t_{0}^{\prime}\right)= & \frac{i}{2 \pi \sqrt{\Phi_{c} \Phi_{d}}} \int_{-\infty}^{\infty} m_{\mathrm{o}}\left(t_{2}^{\prime}\right) \exp \left(\frac{i t_{2}^{\prime 2}}{2 \gamma}\right) \\
& \times \exp \left[-i \frac{\left(t_{2}^{\prime}-t_{0}^{\prime}\right)^{2}}{2 \Phi_{c}}-i \frac{\left(t_{2}-t_{2}^{\prime}\right)^{2}}{2 \Phi_{d}}\right] d t_{2}^{\prime} .
\end{aligned}
$$


Employing Eq. (5), the two-time mutual coherence function at the detectors is expressible as

$$
\Gamma\left(t_{1}, t_{2}\right)=\iint_{-\infty}^{\infty} \Gamma_{0}\left(t_{0}, t_{0}^{\prime}\right) K_{1}^{*}\left(t_{1}, t_{0}\right) K\left(t_{2}, t_{0}^{\prime}\right) d t_{0} d t_{0}^{\prime},
$$

where $\Gamma_{0}\left(t_{0}, t_{0}^{\prime}\right)$ is the coherence function of the source field. The coherence function representing the temporally incoherent source can be approximated as

$$
\Gamma_{0}\left(t_{0}, t_{0}^{\prime}\right)=I_{0} \delta\left(t_{0}^{\prime}-t_{0}\right)
$$

where $I_{0}$ describes the source intensity and $\delta\left(t_{0}^{\prime}-t_{0}\right)$ denotes the Dirac delta function.

\section{IMAGING OF TEMPORAL PHASE OBJECTS}

Next we substitute Eqs. (6), (7), and (9) into Eq. (8) and perform the integrations with respect to $t_{0}^{\prime}$ and $t_{0}$. We additionally assume two conditions:

Condition ( $i$ ). The GDD parameters are taken to satisfy the temporal analog of the spatial lensless ghost-imaging condition, viz., $\Phi_{c}=\Phi_{a}+\Phi_{b}$. The above procedure then leads to

$$
\begin{aligned}
\Gamma\left(t_{1}, t_{2}\right)= & \sqrt{\frac{i I_{0}^{2}}{(2 \pi)^{3} \Phi_{b}^{2} \Phi_{c}} \iint_{-\infty}^{\infty} m_{1}^{*}\left(t_{1}^{\prime}\right) m\left(t_{2}^{\prime}\right) \exp \left(\frac{i t_{2}^{\prime 2}}{2 \gamma}\right)} \\
& \times \exp \left[i \frac{\left(t_{1}^{\prime}-t_{1}\right)^{2}-\left(t_{1}^{\prime}-t_{2}^{\prime}\right)^{2}}{2 \Phi_{b}}\right] \\
& \times \exp \left[-i \frac{\left(t_{2}-t_{2}^{\prime}\right)^{2}}{2 \Phi_{d}}\right] d t_{1}^{\prime} d t_{2}^{\prime} .
\end{aligned}
$$

Condition (ii). The focal length $\gamma$ satisfies the lens equation:

$$
\frac{1}{\gamma}=\frac{1}{\Phi_{b}}+\frac{1}{\Phi_{d}}
$$

On combining Eqs. (10) and (11) with Eq. (2), the correlation between the intensity fluctuations at the detectors can be expressed as

$$
\begin{aligned}
& \left\langle\Delta I_{1}\left(t_{1}\right) \Delta I_{2}\left(t_{2}\right)\right\rangle_{\text {int }} \\
& \quad=A \int_{-T}^{T}\left|\int_{-\infty}^{\infty} m^{\prime}\left(t_{2}^{\prime}\right) \tilde{m}_{1}^{*}\left(t_{2}^{\prime}-t_{1}\right) d t_{2}^{\prime}\right|^{2} d t_{2},
\end{aligned}
$$

where $A=I_{0}^{2} /(2 \pi)^{3} \Phi_{b}^{2}\left|\Phi_{c}\right|$ is a constant containing various system parameters. In addition, we introduced

$$
m^{\prime}\left(t_{2}^{\prime}\right)=m\left(t_{2}^{\prime}\right) \exp \left(\frac{i t_{2} t_{2}^{\prime}}{\Phi_{d}}\right)
$$

and

$$
\tilde{m}_{1}\left(t_{2}^{\prime}-t_{1}\right)=\int_{-\infty}^{\infty} m_{1}\left(t_{1}^{\prime}\right) \exp \left[\frac{-i\left(t_{2}^{\prime}-t_{1}\right) t_{1}^{\prime}}{\Phi_{b}}\right] d t_{1}^{\prime} .
$$

The above expression is the Fourier transform of the mask transmission function. The right-hand side of Eq. (12) is thus simply an integral over the squared modulus of the convolution of functions $m^{\prime}$ and $\tilde{m}_{1}^{*}$.

The ghost-imaging setup works as a temporal analog of the conventional spatial 4- $f$ coherent image processing system [29]. The 4- $f$ scheme consist of two lenses forming a pair of Fourier transforming subsystems with a mask placed at

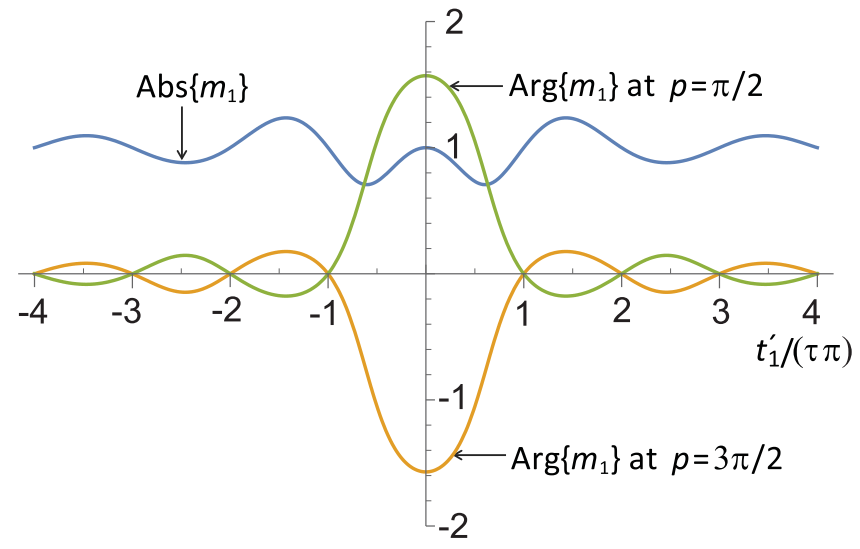

FIG. 2. The absolute value (blue line) and phase (green line for $p=\pi / 2$ and brown line for $p=3 \pi / 2$ ) of the modulation function $m_{1}\left(t_{1}^{\prime}\right)$.

the Fourier plane of the lenses. The intensity at the image plane of the system is proportional to the convolution of the amplitude transmittance function of the object and the Fourier transform of the mask's transmission function. This leads to an expression similar to Eq. (12) with $m^{\prime}\left(t_{2}^{\prime}\right)$ and $m_{1}^{*}\left(t_{2}^{\prime}-t_{1}\right)$ corresponding to the amplitude transmittances of the object and the mask, respectively. Therefore, we may regard $m_{1}^{*}\left(t_{1}^{\prime}\right)$ as the temporal ghost-imaging counterpart of the Fourier-space mask of the traditional $4-f$ system. The mask affects the image properties by suitably selecting and processing the Fourier components transmitted to the image plane. Analogously, in the case of the temporal ghost imaging scheme it is possible to choose the properties of the temporal filter to control the quality of the resultant ghost image.

In Zernike's phase-contrast imaging, the phases of the spatial-frequency components must be shifted within a certain localized window function [22]. Here, for mathematical convenience, we choose the phase-shifting function of the form

$$
m_{1}\left(t_{1}^{\prime}\right)=1-[1-\exp (i p)] \operatorname{sinc}\left(\frac{t_{1}^{\prime}}{\tau}\right)
$$

where $p \in\{\pi / 2,3 \pi / 2\}, \tau$ denotes the duration of the reference-arm modulation, and $\operatorname{sinc}(x)=\sin (\pi x) / \pi x$ is the normalized sinc function. The absolute value and phase profiles of $m_{1}\left(t^{\prime}\right)$ are illustrated in Fig. 2, which shows that $m_{1}\left(t^{\prime}\right)$ performs a phase change of $p$ around $t^{\prime}=0$ but does not significantly affect the amplitude. Even though this is a particular but rather arbitrary choice for $m_{1}\left(t^{\prime}\right)$, the results derived using it will be qualitatively consistent with those obtained by other localized phase-shifting functions. In practice, taking into account the fact that the signal to be modulated is chirped, the modulation can be done by splitting the pulse into spectral components using a diffraction grating, phase-shifting them by $p$ in the central part of the spectrum, and combining all the components back using another grating. 
Using Eqs. (14) and (15), the Fourier transform of the filter function can be expressed as

$$
\begin{aligned}
\tilde{m}_{1}\left(t_{2}^{\prime}-t_{1}\right)= & 2 \pi\left|\Phi_{b}\right| \delta\left(t_{2}^{\prime}-t_{1}\right) \\
& -\tau[1-\exp (i p)] \operatorname{rect}\left[\frac{\tau\left(t_{2}^{\prime}-t_{1}\right)}{2 \pi \Phi_{b}}\right],
\end{aligned}
$$

where $\operatorname{rect}(x)$ denotes the rectangle function. Inserting Eq. (16) into Eq. (12) and performing the integration over $t_{2}^{\prime}$, the correlation function of the intensity fluctuations can be written as

$$
\begin{aligned}
\left\langle\Delta I_{1}\left(t_{1}\right) \Delta I_{2}\left(t_{2}\right)\right\rangle_{\text {int }}^{( \pm)} & \\
= & \frac{I_{0}^{2}}{2 \pi\left|\Phi_{c}\right|} \int_{-T}^{T}\left\{1-2\left[1 \mp \Delta \phi\left(t_{1}\right)\right] \operatorname{sinc}\left(\frac{\Phi_{b} t_{2}}{\Phi_{d} \tau}\right)\right. \\
& \left.+2 \operatorname{sinc}^{2}\left(\frac{\Phi_{b} t_{2}}{\Phi_{d} \tau}\right)\right\} d t_{2},
\end{aligned}
$$

where the upper and lower signs correspond to $p=\pi / 2$ and $p=3 \pi / 2$, respectively. Next we assume that the measurement time is sufficiently long so $T \gg\left|\Phi_{d} \tau / \Phi_{b}\right|$ is satisfied. This allows us to extend the integration limits to infinity in the sinc integrations, which results in

$$
\left\langle\Delta I_{1}\left(t_{1}\right) \Delta I_{2}\left(t_{2}\right)\right\rangle_{\mathrm{int}}^{( \pm)}=\frac{I_{0}^{2}}{\pi\left|\Phi_{c}\right|}\left[T \pm \Delta \phi\left(t_{1}\right)\left|\frac{\Phi_{d}}{\Phi_{b}}\right| \tau\right],
$$

where we used the fact that $\tau>0$. Clearly, the correlation between the intensity fluctuations measured by the photodiodes is proportional to the phase variation induced by the object. The result in Eq. (18) is valid for temporally incoherent light source obeying Gaussian statistics when requirements (i) and (ii) hold.

The object's phase variation can be extracted from Eq. (18), for example, by subtracting the two correlation functions as

$$
C\left(t_{1}\right)=\left\langle\Delta I_{1}\left(t_{1}\right) \Delta I_{2}\left(t_{2}\right)\right\rangle_{\text {int }}^{(+)}-\left\langle\Delta I_{1}\left(t_{1}\right) \Delta I_{2}\left(t_{2}\right)\right\rangle_{\text {int }}^{(-)},
$$

which implies

$$
C\left(t_{1}\right)=\frac{2 I_{0}^{2} \tau}{\pi}\left|\frac{\Phi_{d}}{\Phi_{c} \Phi_{b}}\right| \Delta \phi\left(t_{1}\right) .
$$

The normalized temporal phase variation $\Delta \phi\left(t_{1}\right)$ can thus be retrieved by measuring $\left\langle\Delta I_{1}\left(t_{1}\right) \Delta I_{2}\left(t_{2}\right)\right\rangle_{\text {int }}^{(-)}$and $\left\langle\Delta I_{1}\left(t_{1}\right) \Delta I_{2}\left(t_{2}\right)\right\rangle_{\text {int }}^{(+)}$, corresponding to the masks with opposite phase contrast.

\section{CONCLUSIONS}

We designed a ghost-imaging setup for phase-contrast imaging of pure phase signals in the time domain. The device relies on the use of a temporally incoherent source obeying Gaussian statistics and the measurement of intensity correlations between a fast and a slow photodetector. In general, the intensity correlation function is found to carry information analogous to that of the conventional spatial 4$f$ coherent imaging scheme with a suitable phase mask in the Fourier plane. Choosing the transmission function of the temporal mask in the reference arm suitably, the temporal phase variation in the signal may be converted to a measurable modulation in the retrieved intensity correlation function. The introduced technique is well suited for the characterization of rapidly varying temporal phase signals even when the waveform has been distorted during the transmission, and may find applications in communications and remote sensing.

\section{ACKNOWLEDGMENTS}

This research was funded by the Academy of Finland (Projects No. 308393, No. 308394, and No. 310511). A.H. acknowledges financial support from the Emil Aaltonen Foundation. This work is part of the Academy of Finland Flagship Programme, Photonics Research and Innovation (PREIN, 320166).
[1] D. S. Simon, G. Jaeger, and A. V. Sergienko, Quantum Metrology, Imaging, and Communication (Springer, New York, 2017), Chap. 6.

[2] M. J. Padgett and R. W. Boyd, An introduction to ghost imaging: quantum and classical, Philos. Trans. R. Soc. A 375, 20160233 (2017).

[3] B. J. Hoenders, Review of a bewildering classical-quantum phenomenon: Ghost imaging, in Advances in Imaging and Electron Physics, edited by P. W. Hawkes (Elsevier, Amsterdam, 2018), Vol. 208, pp. 1-41.

[4] P. B. Dixon, G. A. Howland, K. W. C. Chan, C. O'SullivanHale, B. Rodenburg, N. D. Hardy, J. H. Shapiro, D. S. Simon, A. V. Sergienko, R. W. Boyd, and J. C. Howell, Quantum ghost imaging through turbulence, Phys. Rev. A 83, 051803(R) (2011).

[5] R. E. Meyers, K. S. Deacon, and Y. Shih, Turbulence-free ghost imaging, Appl. Phys. Lett. 98, 111115 (2011).

[6] M. Bina, D. Magatti, M. Molteni, A. Gatti, L. A. Lugiato, and F. Ferri, Backscattering Differential Ghost Imaging in Turbid Media, Phys. Rev. Lett. 110, 083901 (2013).
[7] Y.-K. Xu, W.-T. Liu, E.-F. Zhang, Q. Li, H.-Y. Dai, and P.-X. Chen, Is ghost imaging intrinsically more powerful against scattering? Opt. Express 23, 32993 (2015).

[8] P. A. Morris, R. S. Aspden, J. E. C. Bell, R. W. Boyd, and M. J. Padgett, Imaging with a small number of photons, Nat. Commun. 6, 5913 (2015).

[9] A. Meda, A. Caprile, A. Avella, I. Ruo Berchera, I. P. Degiovanni, A. Magni, and M. Genovese, Magneto-optical imaging technique for hostile environments: The ghost imaging approach, Appl. Phys. Lett. 106, 262405 (2015).

[10] D. Pelliccia, A. Rack, M. Scheel, V. Cantelli, and D. M. Paganin, Experimental X-Ray Ghost Imaging, Phys. Rev. Lett. 117, 113902 (2016).

[11] R. I. Khakimov, B. M. Henson, D. K. Shin, S. S. Hodgman, R. G. Dall, K. G. H. Baldwin, and A. G. Truscott, Ghost imaging with atoms, Nature 540, 100 (2016).

[12] A. M. Kingston, G. R. Myers, D. Pelliccia, F. Salvemini, J. J. Bevitt, U. Garbe, and D. M. Paganin, Neutron ghost imaging, Phys. Rev. A 101, 053844 (2020). 
[13] L. Wang, S. Zhao, W. Cheng, L. Gong, and H. Chen, Optical image hiding based on computational ghost imaging, Opt. Commun. 366, 314 (2016).

[14] P. Janassek, S. Blumenstein, and W. Elsässer, Ghost Spectroscopy with Classical Thermal Light Emitted by a Superluminescent Diode, Phys. Rev. Appl. 9, 021001 (2018).

[15] A. Hannonen, A. T. Friberg, and T. Setälä, Classical spectral ghost ellipsometry, Opt. Lett. 41, 4943 (2016).

[16] A. Hannonen, A. T. Friberg, and T. Setälä, Classical ghostimaging spectral ellipsometer, J. Opt. Soc. Am. A 34, 1360 (2017).

[17] A. Hannonen, B. J. Hoenders, W. Elsässer, A. T. Friberg, and T. Setälä, Ghost polarimetry using Stokes correlations, J. Opt. Soc. Am. A 37, 714 (2020).

[18] T. Setälä, T. Shirai, and A. T. Friberg, Fractional Fourier transform in temporal ghost imaging with classical light, Phys. Rev. A 82, 043813 (2010).

[19] T. Shirai, T. Setälä, and A. T. Friberg, Temporal ghost imaging with classical non-stationary pulsed light, J. Opt. Soc. Am. B 27, 2549 (2010).

[20] P. Ryczkowski, M. Barbier, A. T. Friberg, J. M. Dudley, and G. Gently, Ghost imaging in the time domain, Nat. Photon. 10, 167 (2016).
[21] P. Ryczkowski, M. Barbier, A. T. Friberg, J. M. Dudley, and G. Gently, Magnified time-domain ghost imaging, APL Photon. 2, 046102 (2017).

[22] J. W. Goodman, Introduction to Fourier Optics (Roberts \& Company, Englewood, CO, 2005).

[23] T. Shirai, Modern aspects of intensity interferometry with classical light, in Progress in Optics, edited by T. D. Visser (Elsevier, Amsterdam, 2017), Vol. 62, pp. 1-72.

[24] T. Shirai, T. Setälä, and A. T. Friberg, Ghost imaging of phase objects with classical incoherent light, Phys. Rev. A 84, 041801(R) (2011).

[25] E. Ilina, M. Nyman, I. Svagzdyte, N. Chekurov, M. Kaivola, T. Setälä, and A. Shevchenko, Aberration-insensitive microscopy using optical field-correlation imaging, APL Photon. 4, 066102 (2019).

[26] I. A. Walmsley and C. Dorrer, Characterization of ultrashort electromagnetic pulses, Adv. Opt. Photon. 1, 308 (2009).

[27] B. H. Kolner and M. Nazarathy, Temporal imaging with a time lens, Opt. Lett. 14, 630 (1989).

[28] L. Mandel and E. Wolf, Optical Coherence and Quantum Optics (Cambridge University Press, Cambridge, 1995).

[29] B. E. A. Saleh and M. C. Teich, Fundamentals of Photonics (Wiley, Hoboken, 2007).

[30] B. H. Kolner, Space-Time Duality and the Theory of Temporal Imaging, IEEE J. Quantum Electron. 30, 1951 (1994). 